\title{
Rice Consumption Is Associated with Better Nutrient Intake and Diet Quality in Adults: National Health and Nutrition Examination Survey (NHANES) 2005-2010
}

\author{
Theresa A. Nicklas' ${ }^{1}$, Carol E. O’Neil'², Victor L. Fulgoni ${ }^{3}$ \\ ${ }^{1}$ Baylor College of Medicine, USDA/ARS Children's Nutrition Research Center, Houston, USA \\ ${ }^{2}$ Louisiana State University Agricultural Center, Baton Rouge, USA \\ ${ }^{3}$ Nutrition Impact, LLC, Battle Creek, USA \\ Email: tnicklas@bcm.edu, $\underline{\text { CEOneil@agcenter.Isu.edu, vic3rd@aol.com }}$
}

Received 20 August 2013; revised 20 September 2013; accepted 27 September 2013

Copyright $@ 2014$ by authors and Scientific Research Publishing Inc.

This work is licensed under the Creative Commons Attribution International License (CC BY). http://creativecommons.org/licenses/by/4.0/

(c) (;) Open Access

\section{Abstract}

Objectives: The goal of this study was to determine the association of rice consumption with nutrient intake and diet quality in a recent nationally representative sample of US adults. Methods: NHANES data (2005-2010) were used to assess the association of rice consumption by adults (19+ yrs; $N=14,386$ ) with nutrient intake and diet quality. 24-hour dietary intakes were used to calculate usual intake (UI) of rice consumption using the National Cancer Institute methodology. Rice consumption groups were $<0.25,>0.25$ to $<0.5,>0.5$ to $<1.0$, and $>1.0 \mathrm{oz}$. eq. of UI of rice. Diet quality (using the Healthy Eating Index-2005 [HEI-2005]) was calculated. Covariate adjusted least square means \pm SE were determined and quartile trends across the rice consumption categories were examined. Results: Significant $(p<0.001)$ positive trends $(\beta$ coefficient across rice consumption categories) were seen for sodium $(118.99 \mathrm{mg})$, dietary fiber $(0.57 \mathrm{~g})$, folate $(58.24 \mu \mathrm{g} \mathrm{DFE})$, magnesium (11.83 $\mathrm{mg}$ ), iron $(0.97 \mathrm{mg}$ ) and potassium $(29.45 \mathrm{mg})$. Significant negative trends (p < $0.0001)$ were seen for intakes of saturated fatty acids $(-1.75 \mathrm{~g})$, added sugars $(-1.31 \mathrm{~g})$; and calcium $(-40.46 \mathrm{mg})$. HEI-2005 also showed a positive trend $(p<0.0001)$ with rice consumption $(5.5$ points). HEI-2005 component scores for total fruit $(0.07)$, whole fruit $(0.11)$, dark green/orange vegetables $(0.25)$, total grains $(0.10)$, meat/beans $(0.24)$, and oils $(0.15)$ were higher $(p<0.01)$ in rice consumers than non-consumers. HEI-2005 component scores for saturated fatty acids (0.63), solid fats, added sugars, and alcohol (1.22) were higher suggesting more favorable intake, but sodium $(-\mathbf{0 . 2 4})$ was lower. Conclusion: Overall, consumption of rice should be encouraged to improve nutrient intake and diet quality. Nutrition education can provide ways to reduce sodium added to rice dishes. 


\section{Keywords}

\section{Rice Consumption; NHANES; Adults; Nutrient Intake; Diet Quality}

\section{Introduction}

White rice is a refined grain that is a staple food in many parts of the world and is becoming more widely consumed in the US [1]. Brown rice is a whole grain but is less widely consumed than enriched, fortified white rice. Rice consumption has doubled in the US over the last 20 years [2]. Current intake of rice in the US is approximately 21.2 pounds per captia/year [3], with more than $70 \%$ being enriched, fortified white rice. This may be the result from a public emphasis on healthy lifestyles, the rising demand for gluten-free foods, or continued introduction and domestic use of new rice-based products [4].

Demographic factors including socioeconomic, immigration, and changing ethnic composition with groups with high per capita rice consumption, including Latinos and Asians, have also been associated with higher intakes of rice [1]. Race/ethnicity is an especially important influencing factor. The NHANES 2001-2002 data were used to show that Asians and Asian-Americans had the highest consumption of rice in the US [1], confirming results from a previous study using data from a smaller population in Houston, Texas [5]. The NHANES data showed that $48.3 \%$ of "other" races/ethnicities (including Asians, Pacific Islanders, American Indians, and Alaskan Natives) consumed at least $1 / 4$ cup of cooked rice/day, compared with only $13.4 \%$ of non-Hispanic whites, $16.5 \%$ of non-Hispanic blacks, and 21.8\% of Mexican-Americans [1]. "Non-white groups" have been shown in other studies [1] to consume more rice than non-Hispanic whites. Further clarification of rice consumption patterns is clearly needed, since Goodwin et al. [5] found different levels of consumption among different Asian-American groups.

Refined grains, the overwhelming majority of which are enriched, constitute the majority of grains consumed in the US. Children, 1 - 3 years of age (y) and 4 - 8 y consumed 3.9 and 6.1 ounce equivalents (oz. eq.) of nonwhole grains/day. Males and females 9 - 13 y and 14 - 18 y consumed 7.7, 8.5, 6.4, and 6.1 oz. eq., respectively, of non-whole grains/day. Adult males and females 19+ y consumed 7.2 and 5.3 oz. eq., respectively. When considering those $2+\mathrm{y}$, yeast breads (25.9\%), pizza (11.4\%), and grain based desserts $(9.9 \%)$ were the most commonly consumed refined grain products [6]. Rice and rice mixed dishes constituted only $4.4 \%$ of refined grain products consumed [6]. These data suggest that many refined grains are a part of dishes that are high in saturated fatty acids (SFA), solid fats, and sodium [7]; however, this may not the case for rice and most mixed dishes made from rice.

Enriched, fortified white rice contributes more than 15 vitamins and minerals, including folate and other Bvitamins, iron, and zinc to the diet; it is sodium and cholesterol free, and has only a trace of fat, with no SFA [8]. It has been argued that although some vitamins and minerals stripped from grains during the refining process are added back during the enrichment process (iron, thiamin, riboflavin, niacin, and folic acid), not all vitamins are replaced and dietary fiber is not replaced [6]. Brown rice is slightly higher in dietary fiber, magnesium, and phosphorus than enriched, fortified white rice, but it is lower in B vitamins and iron since it is not enriched [8]. Thus, the effect of consuming rice on nutrient intake is unclear and few studies have looked at both types of rice.

Fulgoni et al. [9] showed, using data from the NHANES 1999-2004, that those consuming at least 1 daily serving (approximately 1/4 cup) of rice (enriched, fortified white [refined] or brown) consumed less total fat, SFA, and added sugars than non-consumers. Rice consumers also had higher intakes dietary fiber [1] and of more than 12 essential vitamins and minerals, including iron [1] [9], folate, and other B vitamins [9], and potassium [1] [9], and consumed nearly 4 tsp. (16 g) less added sugar and $7 \mathrm{~g}$ less solid fats than non-consumers [9]. These data suggest that rice consumption makes a positive contribution to nutrient intake beyond the actual nutrient composition of rice. This suggests that rice consumers have healthier lifestyles and make better overall dietary choices than non-consumers.

Sun et al. [10] showed that brown rice consumption was associated with a more health-conscious lifestyle and dietary profile, including being more physically active, leaner, or less likely to smoke; consumers also had higher intakes of fruit, vegetables, and whole grains and lower intake of red meat and trans fat. Rice consumers also consumed more grains (but not whole grains) [1], fruit [9] [10], vegetables, including legumes [1] [10], and 
fewer potatoes [1].

Despite these few studies, the effect of rice consumption on nutrient intake and diet quality using recent nationally representative data is unknown. The objective of this study was to determine the association of rice consumption with nutrient intake and diet quality in a recent nationally representative sample of US adults.

\section{Subjects and Methods}

\subsection{Study Population and Analytic Sample}

Data from adults 19+ years of age $(y)(n=14,386)$ participating in the NHANES 2005-2006, 2007-2008, and 2009-2010 were combined to increase sample size [11]. Analyses included only individuals with complete and reliable dietary records as determined the National Center for Health Statistics staff and excluded females who were pregnant or lactating. This was a secondary data analysis which lacked personal identifiers; therefore, this study did not require institutional review [12] [13].

Demographic information, including age, gender, race-ethnicity, poverty index ratio (PIR), physical activity levels, and smoking status (current smoker yes/no), used for covariates in the statistical analyses outlined below, was determined via interview [14].

\subsection{Dietary Analyses}

Dietary intake was determined using two multiple pass 24-hour dietary recalls [15]. The first recall was in-person in the Mobile Examination Center and the second was conducted 3 - 10 days later via telephone [16]. The Food Commodity Intake Database (FCID) [17] was used to identify ingredients of survey foods that included rice (white or brown rice). The gram amount of rice consumed by NHANES 2005-2010 respondents was determined by applying the rice composition from FCID to the respondent's 24-hour recall dietary interview data. Usual intake (UI) was determined using the National Cancer Institute method [18]. The MPED translates the amounts of foods consumed into the number of equivalents for the seven MyPyramid major groups and the corresponding subgroups. Rice non-consumers were defined by a UI of less than 1/4 oz. ( 7.0875 grams) per day. Rice consumers were defined by intakes of at least $1 / 4$ oz. per day and were further grouped into three rice consumption groups: $>0.25$ to $<0.5,>0.5$ to $<1.0$, and $>1.0$ oz. eq. of UI of rice. Rice consumption categories used in this study were more conservative estimates than what was previously used in other studies [1] [9]. Also, the use of ounce equivalents (oz. eq.) for the rice consumption categories is within the context of MyPyramid Equivalents Database (MPED) [19]. Adjusted total nutrient intakes were determined using the 24-hour dietary recalls and focused on energy and nutrients to be managed in the diet (i.e. SFA, sodium and added sugars) and nutrients of public health concern and those under-consumed by some groups (i.e. dietary fiber, vitamin D, folate, calcium, magnesium, iron, and potassium) [6]. Alcohol intake (g), which was used as a covariate in the statistical analyses, was also determined via the 24-hour dietary recalls.

Diet quality was calculated using the Healthy Eating Index-2005 (HEI-2005) [20] [21]. Food group standards and the development and evaluation of the HEI-2005 have been previously described [22] [23]. The SAS code used to calculate HEI-2005 scores was downloaded from the Center for Nutrition Policy and Promotion website [24]. Briefly, HEI-2005 was designed to evaluate all of the major MyPyramid food groups and major subgroups and nutrients to manage in the diet. The twelve HEI-2005 components were summed for a total possible score of 100 points.

\subsection{Statistical Analyses}

Sample-weighted data were used in all statistical analyses; and, all analyses were performed using SUDAAN Release 9.0.1 (Research Triangle Institute, Research Triangle Park, NC) to adjust the variance for the complex sample design. For 2005-2010, a 6-year weight variable was created by assigning 1/3 of the 2 year weight for 2005-2006, 2007-2008, and 2009-2010 [11]. The sample-weighted percentages (and standard error of the percentages) of the adults in rice groups were calculated using PROC CROSSTAB of SUDAAN. Least-square means (and the standard errors of the least-square means) were calculated using PROC REGRESS of SUDAAN. Covariates for least-square mean nutrient intakes were gender, age (years), race-ethnicity, socioeconomic status (PIR), physical activity level, smoking status and alcohol intake and energy. Similar covariates were used for HEI but not adjusted for energy intake as the HEI formula is based on 1000 calories. A p value of $<0.05$ was 
considered significant.

\section{Results}

\subsection{Demographic and Lifestyle Characteristics of the Sample}

The percentage of adults $19+$ years and older $(n=14,386)$ consuming rice was $84 \%$ with the majority of rice consumers consuming $\geq 0.25$ and $<0.5 \mathrm{oz}$. eq. per day (59\%). Non-consumers of rice were slightly older, more sedentary, and less likely to report being current smokers or drinking alcohol. The gender and ethnicity distribution was similar across the UI of rice consumption categories (Table 1).

\subsection{Association between UI Rice Consumption Groups and Diet Quality (HEI-2005)}

Significant ( $<<0.0001$ ) trends were seen in diet quality (Figure 1); diet quality scores increased 5.5 points between the non-consumers of rice and the highest rice consumers $(\geq 1 \mathrm{oz}$. eq.). Significant positive trends $(\mathrm{p}<$ 0.01 ) ( $\beta$ coefficient across rice categories) were seen for total fruit (0.07), whole fruit $(0.11)$, dark green and orange vegetables (0.25), total grains (0.10), meat and beans (0.24), and oils (0.15). A significant $(\mathrm{p}<0.0001)$ negative trend was seen for milk (-0.36) across the rice consumption categories. HEI-2005 component scores for SFA (0.63), solid fats, alcohol and added sugars (SoFAAS) (1.22) were higher suggesting more favorable intake, but sodium $(-0.24)$ was lower (Table 2$)$.

\subsection{Association between UI Rice Consumption Groups and Nutrient Intakes}

Significant positive trends ( $\beta$ coefficient across rice categories) were seen for adjusted intakes of sodium (118.99,

Table 1. Demographic and lifestyle characteristics of the adults aged $19+$ years ${ }^{1}$.

\begin{tabular}{|c|c|c|c|c|c|c|c|c|c|c|}
\hline \multirow{3}{*}{ Demographic Variables } & \multirow{2}{*}{\multicolumn{2}{|c|}{$\begin{array}{c}\text { All } \\
(\mathrm{n}=14,386)\end{array}$}} & \multicolumn{8}{|c|}{ Usual Rice Intake (oz. equiv.) } \\
\hline & & & \multicolumn{2}{|c|}{$<0.25(\mathrm{n}=2718)$} & \multicolumn{2}{|c|}{$\begin{array}{c}>=0.25-<0.5 \\
(\mathrm{n}=8090)\end{array}$} & \multicolumn{2}{|c|}{$\begin{array}{l}>=0.5-<1.0 \\
(\mathrm{n}=2324)\end{array}$} & \multicolumn{2}{|c|}{$\begin{array}{c}>=1.0 \\
(\mathrm{n}=1254)\end{array}$} \\
\hline & Mean & SE & Mean & SE & Mean & SE & Mean & $\mathrm{SE}$ & Mean & SE \\
\hline Age, years & 46.69 & 0.34 & 61.56 & 0.63 & 44.20 & 0.32 & 45.14 & 0.49 & 38.58 & 0.62 \\
\hline \multicolumn{11}{|l|}{ Gender, \% } \\
\hline Females & 51.18 & 0.42 & 55.89 & 1.19 & 51.71 & 0.72 & 64.59 & 1.36 & 15.51 & 1.28 \\
\hline \multicolumn{11}{|l|}{ Ethnicity, \% } \\
\hline White & 71.47 & 1.88 & 83.44 & 1.53 & 72.69 & 1.85 & 65.96 & 2.27 & 51.04 & 3.29 \\
\hline African-American & 11.25 & 0.98 & 9.00 & 0.98 & 12.18 & 1.13 & 10.44 & 1.04 & 10.64 & 0.98 \\
\hline Mexican-American & 7.80 & 0.91 & 3.53 & 0.74 & 8.19 & 0.96 & 10.25 & 1.17 & 8.82 & 1.09 \\
\hline Poverty Income Ratio & 3.03 & 0.04 & 2.91 & 0.06 & 3.05 & 0.05 & 3.14 & 0.06 & 2.97 & 0.09 \\
\hline \multicolumn{11}{|c|}{ Physical Activity—Intensity, \% } \\
\hline Sedentary & 25.52 & 0.79 & 36.97 & 1.43 & 23.90 & 0.87 & 23.00 & 1.51 & 19.59 & 1.68 \\
\hline Light & 35.91 & 0.66 & 39.97 & 1.28 & 36.07 & 0.81 & 36.01 & 1.35 & 27.13 & 1.75 \\
\hline Moderate-to-Vigorous & 38.57 & 0.89 & 23.06 & 1.63 & 40.02 & 1.01 & 40.99 & 1.48 & 53.28 & 2.14 \\
\hline \multicolumn{11}{|l|}{ Smoking, \% } \\
\hline Current & 23.35 & 0.69 & 14.94 & 1.22 & 26.97 & 0.96 & 20.16 & 1.55 & 20.39 & 1.51 \\
\hline \multicolumn{11}{|l|}{ Alcohol, g } \\
\hline Yes & 11.18 & 0.44 & 7.61 & 0.66 & 12.33 & 0.52 & 9.84 & 0.74 & 12.50 & 1.22 \\
\hline
\end{tabular}

\footnotetext{
${ }^{1}$ Values are presented as means $\pm \mathrm{SE}$.
} 
Table 2. Association between usual rice intake and Healthy Eating Index-2005 ${ }^{1}$ components in adults aged $19+$ years.

\begin{tabular}{|c|c|c|c|c|c|c|c|c|c|c|c|}
\hline \multirow{3}{*}{ HEI-2005 Components ${ }^{2}$} & \multicolumn{8}{|c|}{ Usual Rice Intake (oz. equiv.) } & \multirow{3}{*}{ Beta } & \multirow{3}{*}{ SE } & \multirow{3}{*}{$\begin{array}{c}\text { Quartile } \\
\text { Trend } \\
\text { p-value }\end{array}$} \\
\hline & \multicolumn{2}{|c|}{$<0.25$} & \multicolumn{2}{|c|}{$\geq 0.25-<0.50$} & \multicolumn{2}{|c|}{$\geq 0.50-<1.0$} & \multicolumn{2}{|c|}{$\geq 1.0$} & & & \\
\hline & $\mathbf{L S M}^{1}$ & SE & LSM & SE & LSM & SE & LSM & SE & & & \\
\hline Total Fruit & 2.24 & 0.07 & 2.15 & 0.04 & 2.27 & 0.07 & 2.41 & 0.08 & 0.07 & 0.03 & 0.0167 \\
\hline Whole Fruit & 1.96 & 0.07 & 1.96 & 0.04 & 2.16 & 0.09 & 2.20 & 0.09 & 0.11 & 0.03 & 0.0031 \\
\hline Total Vegetable & 3.21 & 0.05 & 2.98 & 0.03 & 3.13 & 0.05 & 3.27 & 0.08 & 0.04 & 0.03 & 0.1003 \\
\hline Dark Green and Orange Vegetables & 1.08 & 0.05 & 1.09 & 0.03 & 1.42 & 0.07 & 1.78 & 0.08 & 0.25 & 0.03 & $<0.0001$ \\
\hline Total Grains & 4.35 & 0.04 & 4.06 & 0.02 & 4.39 & 0.03 & 4.49 & 0.04 & 0.10 & 0.02 & $<0.0001$ \\
\hline Whole Grains & 1.26 & 0.05 & 1.03 & 0.03 & 1.24 & 0.06 & 1.16 & 0.05 & 0.02 & 0.02 & 0.3966 \\
\hline Milk & 5.30 & 0.10 & 5.15 & 0.06 & 4.87 & 0.11 & 4.13 & 0.14 & -0.36 & 0.05 & $<0.0001$ \\
\hline Meat and Beans & 7.70 & 0.08 & 8.04 & 0.04 & 8.28 & 0.09 & 8.41 & 0.10 & 0.24 & 0.04 & $<0.0001$ \\
\hline Oils & 5.72 & 0.10 & 5.78 & 0.06 & 5.97 & 0.11 & 6.13 & 0.14 & 0.15 & 0.05 & 0.0068 \\
\hline Saturated Fatty Acids ${ }^{3}$ & 5.73 & 0.12 & 5.44 & 0.07 & 6.35 & 0.09 & 7.44 & 0.14 & 0.63 & 0.04 & $<0.0001$ \\
\hline Sodium & 3.69 & 0.11 & 3.81 & 0.06 & 3.38 & 0.07 & 3.09 & 0.13 & -0.24 & 0.05 & $<0.0001$ \\
\hline $\begin{array}{c}\text { Solid Fats, Alcohol, and Added } \\
\text { Sugars (SoFAAS) }\end{array}$ & 9.68 & 0.20 & 9.03 & 0.12 & 10.96 & 0.17 & 12.90 & 0.22 & 1.22 & 0.09 & $<0.0001$ \\
\hline
\end{tabular}

${ }^{1}$ Values are presented as least-square means \pm SE. ${ }^{2}$ Adjusted for gender, ethnicity, age, socioeconomic status (PIR $0-1.25,1.25-3.5,>=3.25$ ), physical activity level (sedentary, moderate, active), current smoking status, and alcohol consumption. ${ }^{3}$ Reversed scored i.e., the higher the score, the lower the intake points.

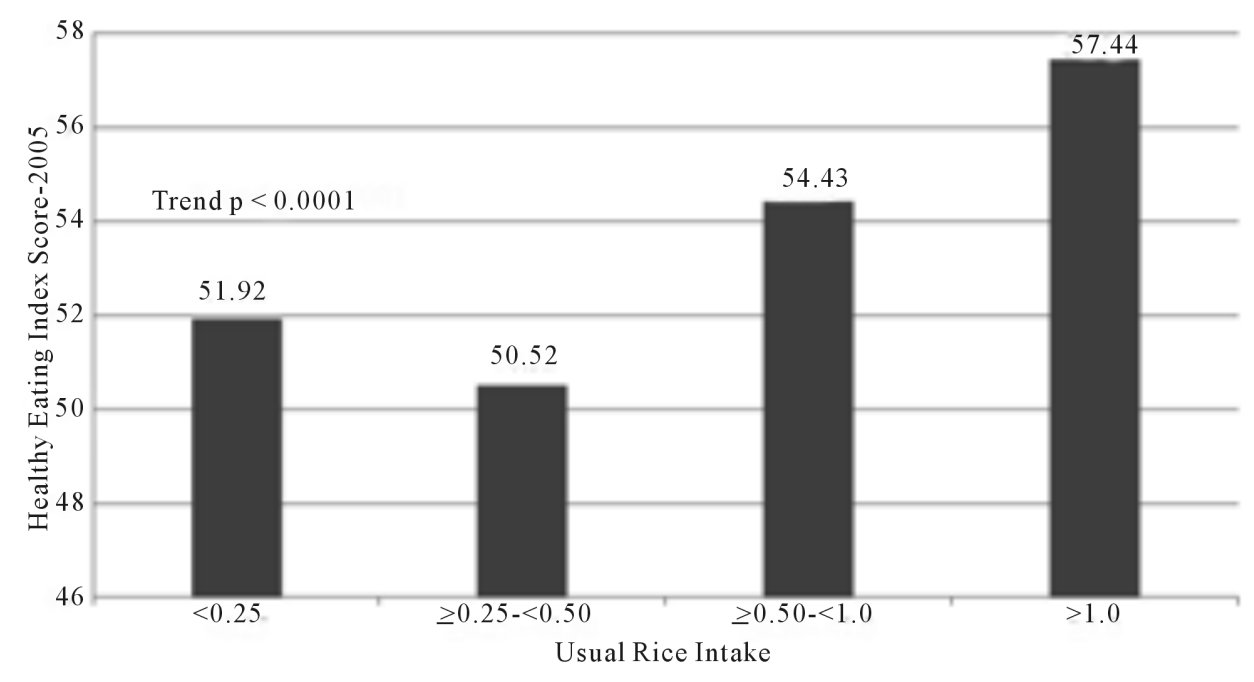

Figure 1. Usual Rice Intake and Diet Quality (HEI-2005). Adjusted for gender, ethnicity, age, socioeconomic status (PIR 0 - 1.25, 1.25 - 3.5, >=3.25), physical activity (sedentary, moderate, active), current smoking status, and alcohol consumption.

$\mathrm{p}<0.0001)$, dietary fiber (0.57, $\mathrm{p}<0.001)$, folate (58.24, $\mathrm{p}<0.0001)$, magnesium $(11.83$, $\mathrm{p}<0.0001)$, iron (0.97, $\mathrm{p}<0.0001)$, and potassium (29.45, $\mathrm{p}<0.05)$. Significant negative trends were seen in intakes of SFA $(-1.75, \mathrm{p}<0.0001)$, added sugars $(-1.31, \mathrm{p}<0.0001)$ and calcium $(-40.46, \mathrm{p}<0.0001)$. There was no association between total energy intakes across the rice consumption categories (Table 3$)$.

\section{Discussion}

These data indicate that rice consumption was associated with better diet quality and nutrient intake than seen in 
Table 3. Association between rice quartiles and intake variables in Adults aged 19+ years participating in the National Health and Nutrition Examination Survey 2005-2010.

\begin{tabular}{|c|c|c|c|c|c|c|c|c|c|c|c|}
\hline \multirow{3}{*}{ Nutrients } & \multicolumn{8}{|c|}{ Usual Rice Intake (oz. equiv.) } & \multirow{3}{*}{ Beta } & \multirow{3}{*}{ SE } & \multirow{3}{*}{$\begin{array}{c}\text { Quartile } \\
\text { Trend } \\
\text { P-value }\end{array}$} \\
\hline & \multicolumn{2}{|c|}{$<0.25$} & \multicolumn{2}{|c|}{$\geq 0.25-<0.50$} & \multicolumn{2}{|c|}{$\geq 0.50-<1.0$} & \multicolumn{2}{|c|}{$\geq 1.0$} & & & \\
\hline & $\mathbf{L S M}^{1}$ & SE & LSM & SE & LSM & SE & LSM & SE & & & \\
\hline \multicolumn{12}{|c|}{ Nutrients to be Managed in the Diet ${ }^{2}$} \\
\hline Energy $(\mathrm{kcal})^{3}$ & 2206.05 & 25.05 & 2127.50 & 15.33 & 2135.93 & 23.31 & 2269.85 & 34.01 & 15.91 & 12.62 & 0.2136 \\
\hline $\begin{array}{l}\text { Saturated Fatty Acids } \\
\qquad(\mathrm{gm})\end{array}$ & 26.92 & 0.33 & 28.15 & 0.18 & 25.92 & 0.17 & 21.79 & 0.45 & -1.75 & 0.14 & $<0.0001$ \\
\hline Sodium (mg) & 3512.84 & 36.32 & 3478.03 & 17.15 & 3610.93 & 25.14 & 3872.53 & 60.24 & 118.99 & 20.06 & $<0.0001$ \\
\hline $\begin{array}{l}\text { Added Sugars (tsp. } \\
\text { equiv.) }\end{array}$ & 18.01 & 0.52 & 18.60 & 0.29 & 16.66 & 0.41 & 14.47 & 0.54 & -1.31 & 0.21 & $<0.0001$ \\
\hline \multicolumn{12}{|c|}{ Nutrients of Public Health Concern or Under-Consumed ${ }^{2}$} \\
\hline Dietary Fiber (gm) & 16.64 & 0.29 & 15.55 & 0.16 & 17.41 & 0.31 & 17.36 & 0.44 & 0.57 & 0.15 & 0.0004 \\
\hline $\begin{array}{l}\text { Vitamin D (D2 + D3) } \\
\text { (mcg) }\end{array}$ & 4.77 & 0.20 & 4.69 & 0.09 & 4.88 & 0.15 & 4.65 & 0.21 & 0.01 & 0.09 & 0.9232 \\
\hline Folate, DFE (mcg) & 538.86 & 8.51 & 501.09 & 5.66 & 614.52 & 11.10 & 673.09 & 14.43 & 58.24 & 5.00 & $<0.0001$ \\
\hline Calcium (mg) & 985.49 & 17.15 & 988.31 & 9.22 & 955.70 & 12.94 & 852.65 & 14.34 & -40.46 & 7.08 & $<0.0001$ \\
\hline Magnesium (mg) & 296.12 & 4.40 & 295.62 & 1.97 & 313.78 & 3.10 & 327.12 & 5.54 & 11.83 & 1.99 & $<0.0001$ \\
\hline Iron (mg) & 15.62 & 0.21 & 14.80 & 0.11 & 16.91 & 0.20 & 17.69 & 0.37 & 0.97 & 0.14 & $<0.0001$ \\
\hline Potassium (mg) & 2721.49 & 30.88 & 2697.61 & 16.24 & 2751.50 & 26.66 & 2792.98 & 41.22 & 29.45 & 13.19 & 0.0305 \\
\hline
\end{tabular}

${ }^{1}$ Values are presented as least square means \pm SE. ${ }^{2}$ Adjusted for gender, ethnicity, age, socioeconomic status (PIR 0 - 1.25, 1.25 - 3.5, >=3.25), physical activity level (sedentary, moderate, active), current smoking status, alcohol consumption, and energy intake. ${ }^{3}$ Adjusted for gender, ethnicity, age, socioeconomic status (PIR 0-1.25, 1.25-3.5, >=3.25), physical activity level (sedentary, moderate, active), current smoking status, and alcohol consumption.

non-rice consumers. These findings are very consistent with a study using earlier NHANES data [9]. The consistency of the association of rice consumption with higher intakes of fiber, folate, magnesium, iron, potassium, SFA, and added sugars is positive. The increase in nutrient intake among rice consumers may reflect the increase in diet quality, specifically the component scores for total fruit, grains, meat and beans, and a decrease in SFA and SoFAAS. A minor difference between this study and the previous study was the association between the oil component score and rice consumption. The conflicting results probably reflect how the oil group was defined. In the current study, the oil group defined in the HEI-2005 [25] was a recommended food group based on the 2005 Dietary Guidelines for Americans [7], whereas, the earlier study used discretionary oils which was a food group to limit in the diet. The increase in total vegetables and grains may reflect an increase in whole grains and dark green and orange vegetables when comparing results from the two studies.

The consistent finding that rice consumers had greater intakes of meat and beans, specifically legumes [9], may in part be due to rice being consumed as a rice and bean mixed dish which is a popular combination food among Hispanics [26] and Southerners [27]. Given that the 2010 Dietary Guidelines for Americans recommend an increase in bean consumption to help meet the current recommendations for dietary fiber, potassium, and magnesium, recommending rice consumption may be a viable strategy to help Americans consume more beans/ legumes in their diet [7].

The negative trend between the milk component score and rice consumption was reflected in a decrease in calcium intake but not potassium intake. This suggests that the increased potassium intake was a result of high fruit and vegetable intake (specifically green and orange vegetables) or bean intake. The milk component in the HEI-2005 [25] is similar to the total dairy food group defined in MyPyramid Equivalent Database [19]. The decrease in the milk component score found in this study may reflect a decrease specifically in cheese consumption among rice consumers and not fluid milk consumption which has been previously reported [9]. This suggests that rice consumers may have different rice consumption patterns that do not incorporate cheese as an ingredient in rice dishes or as a single food. 
Sodium intake was higher in the rice consumption groups. The highest rice consumption group consumed 360 mg more sodium than the non-consumers of rice.

Despite rice being sodium-free, this suggests that some rice products may be processed with added sodium or that consumers season rice dishes with salt or adds high sodium ingredients. However, potassium intake was higher among rice consumers, suggesting that the increased intake of fruits, vegetables, and beans may be helping to negate the sodium intake with additional potassium.

Although findings from this study are consistent with earlier findings [1] [9], one needs to be cautious when comparing the results. Differences in methodologies used (i.e. definitions for determining and categorizing rice consumers; food group definitions; and, years of the studies) varied and need to be considered when interpreting the results. However, the data confirm that rice consumption is associated with positive improvements in the diet. It is important to note that these results are from a cross-sectional epidemiologic study, and as such, one cannot draw conclusions about cause and effect.

Another limitation is the arbitrary definition used to define rice consumers. In this study, and previous studies [1] [9], there was a major assumption that rice consumers were homogenous such that rice consumers had similar rice consumption patterns. Additional analyses showed that six rice consumption patterns were identified using cluster analysis (data not shown). The highest consumption of rice was consumed with meat mixtures and vegetables/legumes. Examination of individual food codes showed that examples of meat mixtures consumed with rice included chicken/beef with or without gravy/sauce; fried rice with meat; or soup with meat. Several of these meat mixtures included vegetables. Several other mixtures included ethnic foods like red beans and rice; shrimp creole; gumbo; jambalaya; chicken chow mein; burrito; or quesadilla. Spanish rice was also a popular rice dish. These preliminary analyses suggest that rice consumption patterns vary considerably. Arbitrary definitions of dietary exposures may be too simplistic of an approach to understanding the relationship between individual foods and overall dietary intakes and diet quality. More studies are needed to better understand the nutritional impact of specific foods in the diet in addition to the assessment of food-based patterns.

\section{Acknowledgements}

This research project was supported by the USDA-Agricultural Research Service through specific cooperative agreement 58-6250-0-008. The contents of this publication do not necessarily reflect the views or policies of the USDA, nor does mention of trade names, commercial products, or organizations imply endorsement from the US government. Partial support was received from the USA Rice Federation and from the USDA Hatch Project LAB 93951. The funding agencies had no input into the study design or interpretation of the data. The authors declare that they have no conflict regarding this paper and have no involvements that might raise the question of bias in the work reported or in the conclusions, implications and opinions stated. Special thanks to Lori Briones for help in preparing the manuscript, and Bee Wong for obtaining research articles.

\section{References}

[1] Batres-Marquez, S.P., Jensen, H.H. and Upton, J. (2009) Rice Consumption in the United States: Recent Evidence from Food Consumption Surveys. Journal of the American Dietetic Association, 109, 1719-1727. http://dx.doi.org/10.1016/j.jada.2009.07.010

[2] Moon, M. (2010) USA Rice: An Update on Commodities and Staple Foods. Nutrition Today, 45, $273-277$. http://dx.doi.org/10.1097/NT.0b013e3181fd4fbe

[3] United States Department of Agriculture. Economic Research Service. Food Consumption and Nutrient Intakes. http://www.ers.usda.gov/Data/FoodConsumption/FoodAvailSpreadsheets.htm\#grains

[4] United States Department of Agriculture. Economic Research Service. Briefing Rooms. Rice Overview. http://www.ers.usda.gov/topics/crops/rice.aspx

[5] Goodwin, H., Holcomb, R. and Rister, M. (1996) A Study of Asian-American Rice Demand in Houston, Texas. Journal of Food Distribution Research, 27, 41-48.

[6] US Department of Agriculture (2010) Center for Nutrition Policy and Promotion. Dietary Guidelines for Americans. http://www.cnpp.usda.gov/DGAs2010-PolicyDocument.htm

[7] Department of Health and Human Services, US Department of Agriculture (2005) Dietary Guidelines for Americans. Washington, DC. http://www.health.gov/dietaryguidelines/dga2005/

[8] United Stated Department of Agriculture. Agricultural Research Service. Nutrient Data Laboratory. 
http://www.nal.usda.gov/fnic/foodcomp/search

[9] Fulgoni III, V., Fulgoni, S., Upton, J. and Moon, M. (2010) Diet Quality and Markers for Human Health in Rice Eaters versus Non-Rice Eaters: An Analysis of the US NHANES, 1999-2004. Nutrition Today, 45, 262-272. http://dx.doi.org/10.1097/NT.0b013e3181fd4f29

[10] Sun, Q., Spiegelman, D., van Dam, R.M., Holmes, M.D., Malik, V.S., Willett, W.C., et al. (2010) White Rice, Brown Rice, and Risk of Type 2 Diabetes in US Men and Women. Archives of Internal Medicine, 170, 961-969. http://dx.doi.org/10.1001/archinternmed.2010.109

[11] National Center for Health Statistics (2006) Analytic and Reporting Guidelines: The National Health and Nutrition Examination Survey (NHANES).

http://www.cdc.gov/nchs/data/nhanes/nhanes_03_04/nhanes_analytic_guidelines_dec_2005.pdf

[12] (2007) NHANES: Is My Survey Information Confidential? http://www.cdc.gov/nhanes/pQuestions.htm

[13] United States Department of Health and Human Services. Office of Extramural Research. National Institutes of Health (2012) NIH Exploratory/Developmental Research Grant Award (R21). http://www.grants.nih.gov/grants/funding/r21.htm

[14] Centers for Disease Control and Prevention. National Center for Health Statistics. National Health and Nutrition Examination Survey (NHANES). Physician Examination Procedures Manual. http://www.cdc.gov/nchs/data/nhanes/nhanes_03_04/PE.pdf

[15] Moshfegh, A.J., Rhodes, D.G., Baer, D.J., Murayi, T., Clemens, J.C., Rumpler, W.V., et al. (2008) The US Department of Agriculture Automated Multiple-Pass Method Reduces Bias in the Collection of Energy Intakes. The American Journal of Clinical Nutrition, 88, 324-332.

[16] Center for Disease Control and Prevention. National Center for Health Statistics. National Heath and Nutrition Examination Survey (NHANES). NHANES Dietary Interview Component. http://www.cdc.gov/nchs/data/nhanes/nhanes 03 04/dietary 03 04.pdf

[17] US Environmental Protection Agency. What We Eat in America_Food Commodity Intake Database, 2003-2008. http://fcid.foodrisk.org

[18] Usual Dietary Intakes: SAS Macros for Analysis of a Single Dietary Component. http://riskfactor.cancer.gov/diet/usualintakes/macros_single.html

[19] United States Department of Agriculture. Agriculture Research Service. MyPyramid Equivalents Database. http://www.ars.usda.gov/Services/docs.htm?docid=17558

[20] U.S. Department of Agriculture, Center for Nutrition Policy and Promotion. Healthy Eating Index-2005 Development and Evaluation Technical Report Support Files. http://www.cnpp.usda.gov/HealthyEatingIndex-2005report.htm

[21] Kennedy, E., Ohls, J., Carlson, S. and Fleming, K. (1995) The Healthy Eating Index: Design and Applications. Journal of the American Dietetic Association, 95, 1103-1108. http://dx.doi.org/10.1016/S0002-8223(95)00300-2

[22] Guenther, P.M., Reedy, J. and Krebs-Smith, S.M. (2008) Development of the Healthy Eating Index-2005. Journal of the American Dietetic Association, 108, 1896-1901. http://dx.doi.org/10.1016/j.jada.2008.08.016

[23] Guenther, P.M., Reedy, J., Krebs-Smith, S.M., Reeve, B.B. (2008) Evaluation of the Healthy Eating Index-2005. Journal of the American Dietetic Association, 108, 1854-1864. http://dx.doi.org/10.1016/j.jada.2008.08.011

[24] United States Department of Agriculture. Center for Nutrition Policy and Promotion. Calculation of the Healthy Eating Index-2005 Component and Total Scores for a Population, Subpopulation, or Group. www.cnpp.usda.gov/Publications/HEI/HEI-2005/Readme_HEI2005_NHANES0102PopulationScore.doc

[25] United States Department of Agriculture. Center for Nutrition Policy and Promotion. Healthy Eating Index-2005 Components and Standards for Scoring. http://www.cnpp.usda.gov/Publications/HEI/healthyeatingindex2005factsheet.pdf

[26] (2009) California Rice Statistics and Related National and International Data. California Rice Commision-Statistical Report. www.calrice.org/pdf/2009-Statistical-Report-FINAL.pdf

[27] Thompson, J., Onufrak, S., Connell, C., Zoellner, J., Tussing-Humphreys, L., Bogle, M., et al. (2011) Food and Beverage Choices Contributing to Dietary Guidelines Adherence in the Lower Mississippi Delta. Public Health Nutrition, 14, 2099-2109. http://dx.doi.org/10.1017/S1368980011001443 